\title{
Les pâturages naturels du Nord-Cameroun
}

\author{
par A. VAILLANT \\ Ingénieur des Services Agricoles du Cameroun
}

\section{SITUATION GÉOGRAPHIQUE}

La région étudiée se situe dans le Nord-Cameroun, entre la ligne de partage des eaux du bassin de la Benoué au Sud, le Lac Tchad au Nord, le Massif montagneux du Mandara à l'Ouest, et le Logone à l'Est.

Cette zone soudano-sahélienne est comprise du $10^{\circ}$ au $13^{\circ}$ degré de latitude nord et du $14^{\circ}$ au $16^{\circ}$ degré de longitude est. Elle déborde de part et d'autre de cette limite artificielle et forme un triangle de $300 \mathrm{~km}$ de haut (de Binder à Fort-Lamy) et $250 \mathrm{~km}$ à la base (de la longitude de Garoua à la longitude de Ham).

Le Logone et le Chari assurent tant bien que mal le drainage et l'irrigation de cette vaste plaine de $26.000 \mathrm{~km}^{2}$. A la saison des pluies, les eaux envahissent les dépressions et forment de vastes prairies inondées (yaérés) qui conditionnent en saison sèche la production fourragère.

\section{CLIMAT}

Le climat est typiquement tropical, à une seule saison des pluies.

Il comporte cependant deux variantes importantes suivant les zones.

Plaines hautes à climat soudano-sahélien (Diamaré).

Plaines basses (Mora à Fort-Foureau) à climat sahélien.

Nous ne donnons ici que la climatologie de la plaine du Diamaré (Maroua). Celle de Fort-Foureau accuse une plus grande sécheresse $(530 \mathrm{~mm}$ de pluie) avec des extrêmes plus accusés (température et humidité relative).

1. - Pluviométrie du Diamaré (sur 10 ans, 1931-1940).

- Janvier - Février - Mars - Avril, mois très secs. Traces de pluies en Mars - Avril (5 à $10 \mathrm{~mm}$ ).

- Mai : mois pluvieux intermédiaire $(65.2 \mathrm{~mm})$.

- Juin - Juillet - Août - Septembre, mois très pluvieux $(740,5 \mathrm{~mm})$.
- Octobre : mois pluvieux intermédiaire $(33,2 \mathrm{~mm})$.

- Novembre - Décembre : mois très secs.

L'indice des saisons pluviométriques d'Aubreville s'établit à $4-2-6$ (mois très pluvieux, mois intermédiaires, mois secs).

Pluviométrie moyenne pendant une période de 6 ans (1946-1951)

\begin{tabular}{|c|c|c|}
\hline MOIS & $\begin{array}{l}\text { MOYENNE } \\
\text { de la période }\end{array}$ & $\begin{array}{c}\text { NOMBRE } \\
\text { de joURS } \\
\text { de pluie (période) }\end{array}$ \\
\hline Janvier .... & 0,0 & 0 \\
\hline Février ... & 0,0 & 0 \\
\hline Mars & 0,0 & 0 \\
\hline Avrril...... . & 5,5 & 2 \\
\hline Mai & 65,2 & 8 \\
\hline Juin ...... & 107,4 & 8 \\
\hline Juillet. ... . & 163,0 & 14 \\
\hline Août.... & 313,8 & 17 \\
\hline Septembre & 156,3 & 11 \\
\hline Octobre .. & 33.2 & 2 \\
\hline Novembre & 0,3 & 0 \\
\hline \multirow[t]{2}{*}{ Décembre. } & 0,0 & 0 \\
\hline & 844,7 & 62 \\
\hline
\end{tabular}

2. - Température du Diamaré (période de 6 ans, 1945-1950).

Il y a deux maxima de température, en avril $(33 \circ 3)$ et en novembre (28०8).

Moyenne des maxima : 35,3 .

Moyenne des minima : 22,1.

Minimum absolu de température : 1104.

Maximum absolu de température : $45^{\circ} 6$. 
Moyenne de température pendant une période de 10 ans (1941-1950)

\begin{tabular}{|c|c|c|c|}
\hline MOIS : & $\begin{array}{c}\text { MOYENNE } \\
\text { máxim. journ. } \\
\text { (périóte) }\end{array}$ & $\begin{array}{c}\text { MOYENNE } \\
\text { des } \\
\text { minim. journ. } \\
\text { (nériode) }\end{array}$ & $\frac{\begin{array}{c}\text { MOYENNE } \\
\text { des }\end{array}}{\text { maxim. }+ \text { minim }}$ \\
\hline Janvier. . . . & 33,8 & 19,3 & 26,6 \\
\hline Février ... & 35,6 & 20,9 & 28,3 \\
\hline Mars. . . & 38,8 & 24,1 & 31,5 \\
\hline Avril . . . . . & 40,8 & 25,8 & 33,3 \\
\hline Mai .... & 38,4 & 24,5 & 31,5 \\
\hline Juin. . . . . & 35,7 & 23,0 & 29,4 \\
\hline Juillet . & 32,5 & 21,9 & 27,2 \\
\hline Août . . . . . & 30,5 & 21,3 & 25,8 \\
\hline Septembre. & 32,3 & 21,5 & 26,9 \\
\hline Octobre... & 35,5 & 21,5 & 28,5 \\
\hline Novembre & 36,1 & 21,4 & 28,8 \\
\hline Décembre. & 34,0 & 19,5 & 26,8 \\
\hline
\end{tabular}

3. - Humidité relative et tension de la vapeur d'eau du Diamaré (période sur 10 ans).

En dehors de la saison pluvieuse, on enregistre une grande sécheresse de l'air.

\section{IES SOLS}

La plaine tchadienne du Cameroun est formée de sédiments du quaternaire.

Les principales formations de sols sont :

Argiles compactes (peu perméables et peu fertiles);

Argiles à retrait (perméables et fertiles) ;

Alluvions anciennes plus ou moins fertiles suivant la dégradation du sol ;

Alluvions récentes de mayos (rivières) assez fertiles.

L'érosion du sol est surtout active dans le massif du Mandara où le déboisement par' les cultures a détruit tous les boisements. Les feux de graminées sévissent chaque année dans ces massifs et empêchent le reboisement naturel.

Le reboisement de ces massifs pourrait régulariser les crues des fleuves, diminuer l'érosion des terres de la plaine et l'envahissement par les sables des rivières.

Enfin, le lessivage en profondeur agit sur les sols dénudés (arqiles fluentes, latérites).

Il est urgent d'organiser pour les massifs du Mandara et les terres de la plaine des travaux de conservation des sols.

\begin{tabular}{|c|c|c|c|c|c|c|c|c|}
\hline \multirow{2}{*}{ MOIS } & \multirow{2}{*}{$\begin{array}{l}\text { MOYENNE } \\
\text { des maxima } \\
\text { journ. }\end{array}$} & \multirow{2}{*}{$\begin{array}{l}\text { MOYENNE } \\
\text { des minima } \\
\text { journ. }\end{array}$} & \multicolumn{3}{|c|}{ MOYENNE EN 1952} & \multicolumn{3}{|c|}{$\begin{array}{l}\text { TENSION DE VAPEUR } \\
\text { (Moyenne en 1952) }\end{array}$} \\
\hline & & & 7 heures & 13 heures & 19 heures & 7 heures & 13 , heures & 19 heures \\
\hline & $\%$ & $\%$ & & & & & & \\
\hline Janvier.... & 37 & 18 & 38 & 26 & 31 & 9,6 & 12,9 & 12,6 \\
\hline Février. & 31 & 17 & 37 & 30 & 32 & 10,7 & 15,5 & 13,9 \\
\hline Mars... . & 30 & 17 & & 24 & 27 & & 14,9 & 14,4 \\
\hline Avril... & 44 & 17 & 48 & 30 & 35 & 18,6 & 21,1 & 19,9 \\
\hline Mai ........ & 72 & 28 & 73 & 43 & 56 & 24,8 & 25,6 & 25,2 \\
\hline Juin......... & 84 & 38 & 80 & 53 & 61 & 25,0 & 26,8 & 25,7 \\
\hline Juillet ...... & 92 & 52 & 88 & 65 & 77 & 25,6 & 27,4 & 27,6 \\
\hline Août $\ldots \ldots$. & 95 & 62 & 91 & 71. & 85 & 25,4 & 28,0 & 27,6 \\
\hline Septembre & 93 & 52 & 91 & 68 & 80 & 24,9 & 26,8 & 26,7 \\
\hline Octobre...... & 78 & 32 & 76 & 38 & 56 & 22,5 & 20,8 & 22,2 \\
\hline Novembre. & 47 & 21 & 37 & 22 & 30 & 12,0 & 12,4 & 12,7 \\
\hline Décembre.. & 40 & 21 & 32 & 20 & 25 & 8,7 & 9,5 & 9,8 \\
\hline
\end{tabular}

\section{VENTS}

L'harmattan, vent froid et'sec, souffle d'octobre à mars. Il s'échauffe graduellement en février-mars.

La mousson du golfe de Guinée, chaude et humide, souffle d'avril à octobre.

C'est au contact des périodes de transition que se produisent des bourrasques et des orages violents.

Fréquence en $\%$ de l'ensemble des observations : Calme, 82.9 ; N.E., 10.9 ; Est, 0.3 ; SW, 5.4 ; W, 0.5.
Les réserves de reboisement, malgré leur étendue, n'ont joué jusqu'ici qu'un rôle expérimental, eu égard aux réalisations à entreprendre.

Lorsque les mesures de conservation des sols débordent 'l'activité des services techniques en place, elles relèvent davantage d'un organisme de la Conservation des sols.

Il faut que cet organisme puisse disposer de moyens propres, faisant défaut aux services 
techniques ou administratifs, pour assurer, avec leur collaboration, le succès d'un programme général de la conservation des sols.

\section{DÉMOGRAPHIE}

La population est formée de Peuls, Soudanais. Arabes du Tchad.

Elle représente 600.000 habitants pour le pays des plaines (Maroua, Kaélé, Yagoua, Mora, FortFoureau) soit 23 habitants au $\mathrm{km}^{2}$.

Une famille moyenne étant formée de 5 individus, et chaque groupe famillial cultivant environ 3 ha (mil, arachides, coton, riz), on peut faire les estimations suivantes sur les surfaces utilisées :

Surface globale.............. 2.600000 ha.

$1 / 8$ terres cultivées .......... 300.000 ha.

2/10 massifs montagneux ......

terres incultes........... 500.000 ha.

boisements $\ldots \ldots \ldots \ldots \ldots \ldots$.

7/10 herbages ............ 1.800.000 ha.

\section{ÉTENDUE DES PATURAGES}

Les deux tiers de la plaine tchadienne du Cameroun sont formés de pâturages (1.800.000 ha).

Cette surface herbagère se subdivise elle-même en :

900.000 ha mis en culture tous les 3 ou 4 ans dans

les assolements culturaux africains.

450.000 ha environ d'herbages permanents sur la

rive gauche du Logone (flore spéciale des yaérés :

Echinochloa, riz vivace, Hyparrhenia...).

Ces pâturages permanents reçoivent soit les eaux de crue du Logone (yaérés), soit les eaux pluviales dans les parties basses sans écoulements (mares temporaires).

Les yaérés sont beaucoup plus importants sur la rive gauche du Logone.

Les mares temporaires sont surtout abondantes au sud de Bogo (triangle Dargala, Goudoum-Goudoum, Kaya), et au sud de la route de MoulvoudaiYagoua dans les sols dunaires (Moulvoudaï, Kalfu, Yagoua).

450.000 ha de savane plus ou moins boisee et pâturée.

Tous les herbages sont soumis chaque année aux feux courants à une époque plus ou moins tardive.

\section{SURCHARGE DES PATURAGES}

Le troupeau de bovidés s'élève, pour la région des plaines, à :

Diamaré (Maroua, Kaélé, Yagoua).... 205.000

Mora ... . . . . . . . . . . . . . . . . . . . 45.000

Fort-Foureau .............. 77.000
Soit au total 327.000 bovidés environ, auxquels il faut ajouter le bétail de case, conservé dans les villages (chevaux, ânes, moutons, chèvres : 1.000.000).

L'alimentation des chevaux et du petit bétail de case ne pose pas les mêmes problèmes que celui des grands troupeaux de bovidés.

La nourriture des premiers est assurée par des fanes d'arachides, du mil (chevaux), les maigres pâturages avoisinant les villages, les mares d'eau et les bords de rivières (chèvres, moutons), ainsi que le recours aux branchages de's arbres et arbustes en saison sèche.

L'alimentation des bovidés est beaucoup plus importante en volume et en étendue.

Dès que le tapis herbacé est épuisé (décembre), les bovidés transhument vers les dépressions basses et humides, plus ou moins inondées, où une abondante végétation graminéenne, leur apporte les masses de verdure considerables nécessaires à leur subsistance.

La disparition rapide du tapis herbacé en fin de saison des pluies pose la question de la surcharge des pâturages.

Cette surcharge, dans l'état actuel de nos connaissances, est assez difficile à établir pour. la plaine tchadienne du Cameroun.

On peut cependant en observer les effets dégradants sur la végétation et les mises 'à feu qui soulignent l'insuffisance des pâturages.

On évalue à 6 ha la surface de pâturage disponible en saison des pluies, et de 1 à 2 ha la surface disponible en saison sèche.

D'autre part, les mesures prophylactiques du Service vétérinaire, tendant à augmenter les troupeaux, poseront avec plus d'acuité le probleme de la surcharge du bétail.

Cette surcharge se traduit déjà par le faible poids des bêtes sur pied et la qualité corrélative de la viande.

La notion de la surcharge des pâturages s'établit en fonction de la valeur alimentaire du pâturage et nécessite des études systématiques pour chaque pâturage.

En l'absence de ces données, on estime qu'il y a surcharge des pâturages lorsque la surface globale des pâturages à un moment donné de l'année. par rapport au nombre de têtes de bétail nourri, se traduit par une déficience alimentaire de celui-ci. En d'autres termes, le pâturage est insuffisant à nourrir le bétail qui pâture. Il faut alors de plus grandes surfaces pour atteindre le même résultat. Le bétail est obligé de parcourir de plus grandes distances pour atteindre des pâturages souvent éloignés des points d'eau. Cette alimentation plus pauvre, jointe à une fatigue supplémentaire, se 
traduit par une diminution du poids des bêtes.

On a cherché à établir dans ce cas le nombre d'hectares nécessaires par tête de bétail. Les Services d'Élevage de chaque région, qui disposent de troupeaux surveillés, peuvent établir, pour une saison donnée, le nombre d'hectares nécessaires pour le maintien de leurs troupeaux dans de bonnes conditions alimentaires.

Le calcul du nombre d'hectares de pâturage naturel nécessaire par tête de bétail, par le nombre de mois de saison sèche, est tout aussi empirique. En effet, certaines régions possèdent des paturages permanents inondés 'en saison sèche qui faussent la notion des mois secs.

La surcharge du pâturage entraîne non seulement une diminution du poids des bêtes mais une dégradation du pâturage et des sols. On ne trouve plus finalement que des espèces vivaces soumises au climax à feu. La végétation arbustive tend à disparaître sous l'action répétée des feux de brousse et l'on s'achemine vers des savanes arides (Imperata, Aristida, Loudetia, Andropogon pseudapricus Stapf, etc.).

La-mise à feu des pâturages peut cependant être tolérée dans les yaérés au retrait des eaux, en fin de cycle végétatif des graminées, pour renouveler le pâturage par des repousses jeunes, appétées par le bétail.

Ces formations ne présentent pas de boisements susceptibles d'être détruits par le feu, qui n'exerce alors qu'une action de nettoyage des tiges ligneuses inutiles et provoque de nouvelles repousses sur les souches vivaces résistantes au feu.

Il n'y a donc pas, dans ce cas, les mêmes effets de dégradation de la végétation et du sol comme pour les savanes arborées soumises au pâturage.

\section{AMÉLIORATION DES PATURAGES NATURELS}

Le problème consiste à prolonger le plus possible les pâturages de saison des pluies et les pâturages inondés pour faire la soudure avec les pâturages de la saison des pluies suivante. Cet objectif peut etre atteirit :

$1^{\circ}$ Par le drainage des yaérés. La hauteur d'eau en fin de saison des pluies empêche la pâture dans certains yaérés.

$2^{\circ}$ Par l'irrigation des yaérés pour prolonger la période de pâture et éviter la mise à feu prématurée dégradant le sol et la flore agrostologique (1).

(1) Les travaux de drainage et d'irrigation s'entendent dans un programme général de mise en valeur. du bassin du Logone (assolement riz-coton, pâturages). Ils ne seraient pas rentables uniquement pour les pâturages.
30 Par la création de réserves naturelles de pâturages améliorés d'arrière-saison (novembre-décembre-janvier).

Si la qualité du pâturage ne se pose pas pour les mois d'abondance (juin, juillet, août et septembre), elle se pose davantage avec les mois de sécheresse où la surcharge du bétail arrive à faire disparaître rapidement les bonnes espèces et ne laisse subsister que des espèces vivaces ligneuses.

Dans l'état actuel de ma connaissance, les espèces en régression sont, pour le Diamaré : les Pennisetum, plus tendres que les Andropogonees.

On ne trouve plus ces espèces que le long des cours d'eau où le bétail vient les brouter en dernier lieu.

Dans les régions montagneuses de l'Adamawa, où les graminées annuelles ont une période végétative plus longue en raison des pluies, la surcharge du bétail et les mises à feu périodiques de la savane boisée doivent faire disparaitre de nombreuses espèces qu'il serait utile de connaître et de multiplier dans les réserves naturelles.

Dans tous les cas, la multiplication de ces espèces pose le problème des réserves de pâturages.

Dans ces réserves, ne serait admis qu'un nombre déterminé de bovidés, retiré avant que la dégradation du pâturage ait lieu.

Le probleme consiste donc à proportionner les troupeaux aux possibilités des pâturages établis et à permettre l'amélioration des races bovines parallèlement à une amélioration du pâturage.'

$4^{\circ}$ Par un système de culture introduisant la jachère à graminées dans les assolements africains.

On a cherché à différentes reprises à associer l'élevage à l'agriculture pour résoudre le problème de la conservation des sols cultives :

- production de fumier:

- production de fourrage engrais vert.

L'insuffisance ou l'impraticabilité des moyens en milieu africain doit faire rechercher de nouvelles formules plus proches des conditions locales de réalisation, en associant la jachère pâturée aux cultures.

Le succès de celle associálion dépend :

- du milieu de culture;

- du choix approprié des graminées à multiplier sur les sols mis en jachères;

- du système pare-feux établi avec, les sols cultivés.

Les avantages à en attendre seraient :

- Suppression dè la manutention et du transport onéreux des pailles et du furnier dans une agriculture peu évoluée;

- Suppression, dans une certaine mesure, des moyens mecaniques importants (fauchage et enfouissement des engrais verts); 
- Apport en saison sèche au bétail d'un choix de graminées fourragères, assurant également la couverture du sol.

\section{PROGRAMIME DES RECHERCHES POUR L'AMÉLIORATION DES PATURAGES}

La Station expérimentale de Guétalé, en liaison avec la Ferme de Multiplication de Maroua, est shargée de l'étude et de l'amélioration des pâturages naturels dans le Nord-Cameroun.

Le programme des recherches concerne :

10 Etude systématique et biologique des graminées.

$2^{\circ}$ Collection vivante des graminées locales et à introduire.

$3^{\circ}$ Premières multiplications.

$4^{\circ}$ Étude des régions naturelles.

$5^{\circ}$ Étude de l'ensilage des graminées.

Le Génie Rural pourra être chargé des questions intéressant l'irrigation et le drainage des yaérés.

Un programme d'ensemble devrait faire entrer l'assolement des graminées fourragères dans la culture des casiers rizicoles.

\section{COMPOSITION DES PATURAGES NATURELS}

L'alimentation du bétail (bovins, ovins, caprins, chevaux, ânes) est assurée :

- par le tapis herbacé de saison des pluies ;

- par la persistance en saison sèche de pâturages permanents inondés (yaérés de crues et mares temporaires);

- par les branchages des arbres et arbustes coupés en saison sèche.

Au nord et au sud de la latitude moyenne de Maroua (10.34), la proportion des espèces fourragères varie avec le climat et les conditions du milieu.

$\mathrm{Si}$ les espèces annuelles sont les plus intéressantes au point de vue fourrager, les espèces vivaces permettent de prolonger le pâturage en saison sèche et donnent des repousses après la mise à feu.

Nous allons passer en revue la composition de ces diverses formations herbagères :

\section{Le tapis herbacé annuel de saison des pluies.}

En mars-avril, tous les herbages exondés sont desséchés et le petit bétail de case se nourrit exclusivement d'herbes vivaces au bord des mares ou des rivières et des branchages d'arbres et d'arbustes.

Le gros bétail est dans les yaérés où l'épuisement des pâturages et la sécheresse obligeront à la mise à feu pour provoquer de nouvelles repousses des souches vivaces.

Dès les premières pluies de mai, les plantes annuelles du Diamaré germent et forment un tapis herbacé continu qui atteint 5 à $10 \mathrm{~cm}$ à la fin mai.

C'est le Cyperus maculatus Bøeck et le Cyperus rotondus Linné qui donnent les premières pousses vertes broutées par les chèvres et les moutons.

Puis les graminées annuelles se développent partout. La majeure partie du tapis herbacé est formee par le Brachiaria brachylopha Stapf, qui s'étale pendant toute la saison des pluies.

Avec le Brachiaria brachylopha Stapf, on trouve en mélange :

Brachiaria fulva Stapf; $B$. stigmatisata Stapf ;

$B$. regularis Stapf.

Chloris pilosa Schun et Thonn; C. prieuri Kunth ; C. robusta Stapf.

Dactyloctenium aegyptium Beauv.

Digitaria adscendens Henrard; $D$. squamata

Willd ; $D$. turgida Willd.

Eleusine indica Gaertn.

Eriochlod acrotricha Hack ex Thell.

Ischaemum brachyatherum Tenzl. et Hack.

Panicum laetum Kunth ; P. Iongijubatum Stapf.

Paspalum scrobiculatum Linné.

Pennisetum pedicellatum Trin. ; P. subangustum Stapf.

Rhynchelytrum repens C.E. Hubbard.

Dès le 15 juin, le tapis herbacé annuel atteint 10 à $15 \mathrm{~cm}$ et pcut nourrir les troupeaux qui reviennent des lointains pâturages auprès de leurs villages.

De fin juin à fin août, l'herbe est partout tendre et abondante.

En septembre, la plupart des espèces sont en pleine épiaison et perdent leur valeur fourragère. Normalement, le cycle végétatif des annuelles et des Cyperus se termine fin septembre.

Les plantes annuelles s'adjoignent de nombreuses espèces vivaces qui assurent le relais des annuelles jusqu'à la fin novembre.

Ce sont :

Andropogon tectorum Schun.; A. schirensis Hochst.

Echinochloa obtusiflora Stapf; E. pyramidalis Hitchcock.

Hyparrhenia rufa Stapf.

Panicum repens Linné.

Pennisetum purpureum Schum. ; $P$. setaceum Chiov. ; P. ramosum Schweinf.

Rottboellia exaltata Linné.

Saccharum spontaneum Linné; $S$. officinarum.

En novembre, toute graminée qui n'est pas dans les bas-fonds inondés se dessèche.

Les Cyperus et les annuelles ont fini leur cycle en septembre-octobre. 
Le bétail s'ạchemine vers less yaérés (prairies inondées).

Le petit bétail est nourri avec des branchages, les repousses des souches vivaces brûlées, le Paspalum scrobiculatum, les repousses de Panicum repens, etc...

\section{Espèces graminéennes des prairies inondées (yaérés).}

Le gros bétail trouve en saison sèche des réserves fourragères dans les parties basses et inondées où dominent les diverses espèces d'Echinochloa, Saccolepis, vulgairement appelées bourgou. On désigne sous le nom de "bourgoutière " les parties de yaérés particulièrement riches en ces espèces:

Les principales graminées qui caractérisent là flore des yaérés sont :

Echinochloa stagnina P. Beauv. ; E. obtusiflora Stapf ; E. pyramidalis Hitchcock.

Vossia cuspidata Griff.

Róttboellia exaltata Linné.

Saccolepis interrupta Stapf.

Eragrostis gangetica Steud.

Hyparrhenia rufa Stapf.

Panicum dregeanum Stapf; $P$. aphaneuron Stapf ; $P$. anabaptistum Steud.

Le genre Hyparrhenia occupe une place prépondérante dans les yaérés. Il colonise les terres argileuses ou alluvionnaires inondées avec une hauteur de crue atteignant au maximum 60 à $80 \mathrm{~cm}$. Lorsque les terres sont riches, l'Hyparrhenia est très serré, robuste et haut de taille.

En deuxième importance viennent les Echinochloa :

Echinochloa stagnina P. Beauv. Supporte 1 mètre de crue et davantage (mares profondes et riches en humus).

Echinochloa obtusiflora Stapf. Supporte 50 à $70 \mathrm{~cm}$ de crue; indice des bonnes terres fertiles à riz, mais un peu profondes pour les variétés ne supportant pas une grande lame d'eau.

Vossia cuspidata colonise les terres alluvionnaires des îles du Logone où il résiste au courant el supporte 1 mètre d'eau et plus.

Saccolopis interrupta est très localisé, sur terres riches et profondes, souvent en association avec le riz sauvage (pouss). Il supporte une lame d'eau de 50 à $80 \mathrm{~cm}$.

Oryza perennis Maench, ssp. Barthii, se situe entre 25 et $50 \mathrm{~cm}$. Il constitue un indice d'établissement des rizières cultivées à condition que la lame d'eau soit supportée par. les variétés à introduire en culture.

Il est souvent en association avec Echinochloa obtusiflora dans les bonnes terres riches à grande lame d'eau (50 cin).

Dans les terres riches et moins profondes, on le trouve en association avec Vetiveria nigritana.

La présence d'Echinochloa obtusiflora Stapf est toujours un bon indice favorable de la fertilité des sols et de l'établissement des rizières.

Le Vetiveria se trouve en touffes isolées sur des terrains peu inondés (25 à $40 \mathrm{~cm}$ ). Il est souvent associé au riz sauvage.

Eragrostis gangetica se situe sur les plateaux peu inondés ou secs.

Sa présence n"est pas un caractère favorable à l'établissement des rizières ou à la qualité du sol, généralement pauvre:

Enfin, Cyperus rotundus Linné envahit souvent les rizières peu profondes et se situe sur le niveau des. berges avec très faible niveau de crue.

En février-mars, lorsque les yaérés les moins profonds se sont asséchés, on les brûle pour provoquer de nouvelles repousses de souches vivaces.

Les troupeaux s'éloignent alors toujours plus au Nord de l'immense cuvette qui: s'étend dans le Salamat, au Sud de Fort-Foureau.

\section{Arbres et arbustes fourragers de saison sèche.}

Beaucoup d'arbres et d'arbustes constituent en saison sèche (décembre, janvier, "février, mars, avril) une nourriture fraîche d'appoint utilisée surtout pour le petit bétail-conservé dans les villages et pour le bétail transhumant vers les yaérés.

Cette production fourragère arbustive ne forme pas, comme pour la région sahélo-saharienne, le fond de la subsistance du gros bétail.

L'importance des pâturages permanents de saison sèche (yaérés) confère en effet à la plaine tchadienné $\mathrm{du}$ Cameroun une situation exceptionnelle qui lui permet de nourrir et de faire subsister en pleine saison sèche un bétail important, évalué avec les transhumants à plus de 600.000 têtes.

Les arbres et arbustes fourragers apportent cependant, dans les années sèches surtout, une contribution non négligeable.

A noter que beaucoup' d'arbres et d'arbustes fourragers ne sont appétés qu'en période de disette.

Dès que les graminées repoussent, le bétail les délaisse pour une nourriture meilleure. 'C'est pourquoi certaines plantes sont appétées par'le bétail dans une région donnée, alors que dans. d'autres elles sont refusées.'

Les chevaux sont parmi les plus difficiles sur la palatabilité, puis viennent les bovins, les ovins, les caprins et les chameaux. 


\section{PRINCIPALES GRAMINÉES FOURRAGÈrES DU NORD.CAMEROUN}

L'étude des graminées fourragères les plus connues nous a conduit à la reconnaissance de 30 genres représentant 65 espèces.

Cette liste est loin d'être close.

La plaine tchadienne du Cameroun possède en effet une des flores graminéennes les plus riches et les plus variées du Centre Afrique.

\section{Andropogon.}

Plantes annuelles et vivaces surtout intéressantes à l'état jeune ou par les repousses après la mise à feu.

Andropogon pseudapricus Stapf. - Solondo (Foulbé), annuelle, fourragère avant épiaison. Terres argileuses de saison des pluies (hordé), savanes.

Andropogon schirensis Hochst. - Vivace, fourragère à l'état jeune. Savanes arborées.

Andropogon gayanus Kunth. - Gurufo $(f)\left({ }^{*}\right)$. Vivace, fourragère, état jeune et jusqu'à la floraison; repousses après mises à feu. Constitue le fond de la végétation des andropogonées dans les savanes arborées soumises au climax à feu.

\section{Brachiaria.}

Plantes annuelles intéressantes pour l'alimentation du bétail, entrant dans la composition du tapis herbacé de saison des pluies.

La plus abondante est Brachiaria brachylopha Stapf.

Brachiaria brachylopha Stapf. - Koumbo bade (f). Très bonne plante fourragère annuelle. La plus abondante dans le tapis herbacé. Fournit de l'herbe pendant toute la saison pluvieuse. Tous terrains.

Brachiaria fulva Stapf. - Tchotcholiho (f). Plante annuelle fourragère (chèvres, chevaux). Isolée en savane.

Brachiaria regularis Stapf. - Plante annuelle fourragère. Bonnes terres fraiches.

Brachiaria mutica. - Gambarawal (f). Water grass. Plante vivace pour pâturages permanents.

\section{Chloris.}

Plantes annuelles intéressantes du tapis herbacé de saison des pluies. La plus appréciée est Chloris pilosa Schum. et Thonn.

Chloris lamproparia Stapf. - Didina (banana), Djiddereno (f). Fourragère avant épiaison, plante rudérale. Terres argileuses, arides de saison des pluies (hordé).

$\left(^{*}\right)(f)=$ nom foulbé ; $(a)=$ nom arabe.
Chloris pilosa Schum. et Thonn. - Damaüli (f). Très bon fourrage vendu sur les marchés, plante annuelle. Bonnes terres.

Chloris prieurii Kunth. - Bonne fourragère. Bonnes terres légères de saison des pluies. RBA 1934, 126.

Chloris robusta Stapf. - Vivace en grosses touffes. Bords des rivières; terres humides. RBA 1934, 127.

4. Cynodon dactylon Pers. - Chiendent, herbe des Bermudes; herbe des Bahamas; herbe du Diable; Nierhello (f). Plante gazonnante; fixation des sables, terrains de sports, pelouses tondues et roulées. Fâturée par chèvres et moutons. Plante rudérale, bords des chemins, bords des mayos.

5. Dactyloctenium aegyptium Beauv. - Falandé (f). Excellent fourrage vendu sur les marchés. Plante annuelle commune. Grains comestibles. Bonnes terres et abords des villages.

6. Digitaria. - Plantes annuelles du tapis herbacé. La plus connue et la plus appréciée est Digitaria adscendens Henrard. Digitaria adscendens (HBK) Henrard, var criniformis. - Salasiliwal (f). Bonne fourragère annuelle. Bonnes terres. Digitaria gayana Stapf. - Djarendiho (f). Houssmahana (banana). Annuelle, rudérale, fourragère avant épiaison. Terrains sablonneux pauvres.

7. Dinebra retroflexa Panzer. - Plante à sel. Fourragère appétée pour son goût salé.

8. Echinochloa. - Plantes annuelles ou vivaces à grand développement. Toutes les espèces sont bonnes fourragères à l'état jeune ou adulte. Le fourrage est coupé avant épiaison. Les graines sont comestibles.

Echinochloa colona Link est une excellente plante annuelle du tapis herbacé de saison des pluies.

Echinochloa obtusiflora Stapf, et stagnina Beauv. forment le fond des prairies inondées.

Echinochloa pyramidalis se rencontre au bord des mayos (rivières).

Echinochloa colona Link. - Jungle rice (riz sauvage). Très bon fourrage, graines comestibles, variétés cultivées en Egypte. Plante annuelle connue au Tchad. Bonnes terres fraîches ou humides, terres riches et fraîches.

Echinochloa obtusiflora Stapf. - Kayari (f), Sarmana (banana), Aloa (musgum). Plante annuelle fourragere, abondante dans les yaérés. Terres riches inondées, bords de rivières.

Echinochloa pyramidalis Hitchcock. - Antilop grass. Tagal. Moroina (banana). Garambawal (f). Bonne fourragère état jeune. Terres riches, inondées, bords de rivières. 
Echinochloa stagnina P. Beauv. - Bourgou (f). Bonne fourragère, prairies inondées (yaérés) ; enracinement aux nœuds à la décrue ; permet le pâturage de saisonn. sèche dans les bas-fonds inondés. Tiges sucrées (mélasses). Terres riches inondées, mares.

9. Eleusine indica Gaertn. - Sargalde (f). Fourragère état jeune. Annuelle, long cycle. Plante rudérale.

10. Eragrostis. - Plantes annuelles ou vivaces de saison des pluies, fourragères à l'état jeune, broutées surtout par chèvres et moutons.

Eragrostis gangetica Steud. - Guiguida (banana de yagoua), Darkadei (mosgum). Chiendent vivace des bords des mayos, brouté par chèvres et moutons (yagoua).' $\mathrm{Ne}$ supporte pas une lame d'eau épaisse et continue. Se situe sur les bords des zones d'inondation.

Eragrostis linearis Benth. - Sarawal (f). Fourragère, commune. Terres légères, arịdes. Plante rudérale.

Eragrostis squamata Steud. - Tchoukouhello (f). Pâturée. Terres légères. Plante rudérale.

Eragrostis turgida Willd. - Fourragère annuelle. Terres légères, plante rudérale.

11. Eriochloa acrotricha Hach. ex Thell, - Djalbatari (f). Plante annuelle à fort développement, bonne fourragère de saison des pluies. Terrains frais, bord de l'eau.

12. Hyparrhenia. - Plantes vivaces des prairies inondées, fourragères à l'état jeune et surtout par les repousses de saison sèche après mise à feu.

Hyparrhenia chrysargyrea Stapf. - Fourragère à l'état jeune, vivace. Terres riches, savanes arborées.

Hyparrhenia dissoluta C.E. Hubbard, H. ruprechtii Fourn., $H$. anthistiria dissoluta Nees. - Kangaroo grass, Djoldowal (f). Fourragère à l'état jeune, après les premières pluies et la mise à feu ; plante vivace en touffe. Terres argileuses, savane arborée.

Hyparrhenia hirta Stapf. - Fourragère à l'étát jeune. Bonnes terres, savanes arborées.

Hyparrhenia rufa Stapf. - Vivace, fourragère à l'état jeune: Bonnes terres (yaérés).

Hyparrhenia soluta Stapf. - Fourragère à l'état jeune, vivace. Bonnes terres, savanes arborées.

13. Ischaemum brachyatherum Fenzl. et Hack. Fourragère à l'état jeune, vivace. Bonnes terres argileuses.

14. Loudetia togoensis C.E. Hubbard. - Moubarawal (Garoua), Selbo (f) (Maroua). Plante annuelle peu appréciée. Terres argileuses arides.
15. Oryza perennis Maench: spp. O. Barthii $A$ Chev. - Bonne fourragère avant épiaison, recherchée par les chevaux. En mélange avec Echinochloa sta. gnina P. Beauv' dans les yaérés; graines comestibles ; var. à arêtes blanches et à arêtes rouges. Bonnes terres supportant une lame d'eau de 50 à $60 \mathrm{~cm}$.

16. Panicum. - Plante annuelle et vivace présentant de nombreuses espèces bonnes fourragères entrant dans la composition des pâturages annuels. Coupée avant épiaison pour le fourrage (août).

Les meilleures espèces les plus répandues sont : Panicum laetum Kunth, $P$. longijubatum Stapf, P. repens Linné.

Les autres espèces :

Panicum anabaptistum Steud, $P$, aphanoneuron Stapf, $P$. dregeanum Stapf, ne présentent de l'intérêt que par les repousses jeunes.

Panicum anabaptistum Steud. - Sihuko (f). Plante vivace fourragère à l'état jeune. Bonnes terres argileuses.

Panicum aphanoneuron Stapf. - Vivace. Parties inondées, bonnes terres argileuses humides. R.B.A. 1934, 22.

Panicum dregeanum Stapf. - Diwel (banana de Yagoua). Vivace. Bonnes terres argileuses humides. R.B.A. 1934, 22.

Panicum laetum Kunth. - Kamdala (arabe). Bonne fourragère, annuelle, abondante en zone sahélienne. Terres légères. R.B.A. 1934, 23.

Panicum longijubatum Stapf. - Tchabadiwal dombi (f). Annuelle et vivace, bonne fourragère à l'état jeune. Bonnes terres argileuses fraîches.

Panicum repens Linné. - Nierhello (f). Bonne fourragère des bords de mayo. Fixation des sables. Terres légères, limoneuses, humides.

17. Paspalum. - Plantes vivaces appétées par le bétail.

Une espèce introduite (Paspalum, dilatatum).

Une espèce spontanée (Paspalum scrobiculatum).

Paspalum scrobiculatum Linné.-Kombo boueda (f). Annuelle et vivace, fourragère; graines provoquant des troubles digestifs (Nord-Nigeria) d'après Hutch; pâturée en saison sèche par chèvres et moutons. Bonnes terres. R.B.A. 1933, 890 . B.A.C.B. 21, 1930 : 1386-1387.

18. Pennisetum. - Bonnes plantes fourragères avant épiaison, pouvant convenir pour l'ensilage ; graines comestibles; forte végétation et grand pouvoir couvrant ( $P$. purpureum Schum. et $P$. setaceum Chiov.).

Le Pennisetum setaceum Chiov. constitue un bon fourrage résistant en saison sèche sur terrains alluvionnaires frais (hacher les chaumes pour la consommation du bétail). 
Pennisetum pedicellatum Trin. et $P$. subangustum Stapf peuvent être cultivés comme fourrage à faucher. Rendement surtout intéressant la deuxième année de culture (semis naturels).

Pennisetum pedicellatum Trin. - Wuluko (f), Um dufufu (a). Très bon fourrage annuel vendu sur les marchés; couper avant floraison; végétation rapide' (3 à 4 mois); cultivable. Plante annuelle multipliée par semis. Repousse naturellement les années suivantes. Bonnes terres de jardin.

Pennisetum polystachyon Schult. - Mission grass. Bon fourrage dans le jeune âge; multiplication facile par semis ; plante vivace ; spontanée et cultivée dans le Nord-Cameroun. Bonnes terres.

Pennisetum purpureum Schum. - Elephant grass. Herbe à éléphant, Sissongo (Yaoundé). Bon fourrage etat jeune ( $1 \mathrm{~m}$ ) ; ensilage ; grand pouvoir couvrant (jachères) ; multiplication par divisions de souches, boutures boisées et semis; excellente plante de jachère. Envahissante dans les régions forestières. Terres riches.

Pennisetum ramosum Schweinf. - Vivace, fourragère état jeune. Bonnes terres argileuses.

Pennisetum setaceum Chiov. - Foutain grass, Wutalde mayo (f). Très bonne fourragère à l'état jeune ; résistante en saison sèche; grand pouvoir couvrant (jachères) ; vivace. Terres légères fraîches (alluvions de mayo). Multiplication : éclats de souches, drageons.

Pennisetum subangustum Stapf et Hubbard. Waluko (f). Usages et végétation similaires à ceux de $P$. pedicellatum. Plante annuelle. Multiplication par semis. Bonnes terres de jardin.

19. Rhynchelytrum repens C.E. Hubbard. Tricholène, Koual lihedje (f). Plante annuelle pâturée. Sa culture n'a pas donné de résultats intéressants jusqu'à présent parce qu'elle colonise les sols secs, arides et sablonneux, et supporte mal la concurrence avec les autres graminées.

20. Rotboellia exaltata Linné. - Niello (f). Bonne fourragère à tout âge. Cultivée avec Axonopus (pays Mossi), semis serrés et repiquage dans les plantations de mai3. R.B.A. 1933, 852. Bonnes terres fraîches.

21. Saccolepis interrupta Stapf. - Fofouna (banana de Yagoua), Massna (banana), Tirik (mosgum). Très bonne fourragère. Terres riches à grande lame d'eau et indice des bonnes rizières. Enracinement des tiges couchées à la décrue.

22. Saccharum. - Deux espèces de Saccharum très utiles par leur résistance à la saison sèche. Après hachage, les tiges sont consommées par les bêtes; elles constituent d'autre part d'excellents brise-vents et fixent les sables. Peuvent être utilisées comme limites de plantations.

Le $S$. spontaneum Linné a une aire très vaste de culture. Il a donné d'excellents résultats en Afrique du Nord; connu dans le midi de la France sous le nom de Canne de Provence.

Saccharum spontaneum Linné. - Sugar cane. canne de Provence, canne à sucre sauvage, Agardadji (f). Plante extrêmement précieuse par ses nombreux usages : brise-vents, fixation des sables et dunes, résistance en saison sèche, fourragère (hachage des chaumes) en saison sèche.

Mérite une attention particulière des Services de l'Agriculture, Élevage et Forêts (re-afforestation des régions sableuses). Tous terrains, de préférence argileux et frais. R.B.A. 1933, 856. R.B.A. 1927, 381.

Saccharum officinarum Linné. Agardadji (f). Mêmes références que le $S$. spontaneum. Terres argileuses fraîches. R.B.A. 1933, 856.

23. Setaria. - Plantes annuelles ou vivaces du tapis herbacé de saison des pluies, bonnes fourragères : couper avant épiaison. Terres légères ou argilo-sableuses.

Setaria anceps Stapf. - Witcho wandu (f). Fourragère. Terres légères. R.B.A. 1934, 29.

Setaria barbata Kunth. - Gaouri' tchelli (f). Annuelle, fourragère à l'état jeune. Bonnes terres.

Setaria sphacellata Stapf et Hubbard. - Witcho wandu (f). Fourragère vivace. Terres limoneuses. R.B.A. 1934, 30.

Setaria pallidifusca Stapf et Hubb. - Witcho wandu (f). Fourragère annuelle. Terres légères.

24. Sorghum - Deux espèces connues au Cameroun. Fourragères à l'état jeune et susceptibles d'être cultivées pour ensilage. Une espèce cultivée introduite ( $S$. sudanense Stapf).

Sorghum vogelianum Stapf. - Kamerun grass. Bon fourrage à l'état jeune, plante annuelle $2,50 \mathrm{~m}$; graines comestibles, fourragères. Bonnes terres argileuses, humifères. Sert à délimiter les plantations.

Sorghum arundinaceum Stapf $=S$. halepense. Kamerun grass, Tchinguili (f). Graines comestibles. Délimitation des cultures. Valeur fourragère discutée.

25. Sporobolus. - Plante rudérale broutée par chèvres et moutons.

Sporobulus festivus Hochst. - Bubba dubbel (f). Terres humides peu fertiles.

Sporobulus pyramidalis P. Beauv. - Rat's tail grass. - Lemno (f). Appétée à l'état jeune; vivace, bordures de jardins. Terres arides, plante rudérale.

26. Thelepogon elegans. - Roth ex Roem. et Schult. - Karkade (f), Glaigana (banana). Fourra- 
gère à l'état jêne; appréciée par les chevaux. Bonnes terres. R.B.A. 1933, 849.

27. Urochloa. - Plante fourragère intéressante du tapis herbacé de saison des pluies.

Urochloa" lata C.E. Hubbard. - Boboyoho (f). Très bon fourrage, vendu sur les marchés, cultivable, graines comestibles. Bonnes terres de jardins.

Urochloa trichopus Stapf. - Bonnes terres. Bon fourrage des régions subdésertiques, cultivé (Rhodésie).

28. Vetiveria nigritana Stapf. - Sodorndé (f). Repousses surtout de fin de saison sèche, fourragères après mise à feu. Terres argileuses.

29. Vossia cuspidata Griff. - Garambawal (f), Mourotna (banana), Dikna (banana), Marass (mosgum). S'étend par enracinement des tiges sur les terres inondées. Colonise les îles alluvionnaires du Logone où il forme de véritables prairies flottantes. Bon fourrage à l'état vert. Bonnes terres humides ou inondées.

30. Oxythenanthera abyssinica A. Fich. Feuilles fourragères. Bonnes terres fraîches; bordures de mayos et gorges de montagnes. Assez rare en plaine.

\section{PRINCIPAUX ARBRES ET ARBUSTES FOURRAGERS DU NORD-CAMEROUN}

Les arbres et arbustes fourragers constituent, comme nous l'avons vu, une réserve fourragère des plus utiles en saison sèche.

Le nombre et la variété de ces espèces est une assurance en cas de disette.

Les principales familles d'arbres et d'arbustes fourragers, par ordre d'importance, sont les suivantes :

Légumineuses (Cesalpinées; papilionacées, mimosées) - Capparidacées - Combretacées Rhamndcées - Moracées - Tiliacées - Anonacées - Ampelidacées - Bombacées - Rubiacées Asclepiadacées - Anacárdiacées - Bignoniacées - Simarubacées - Ulmacées - Mornigacées Euphorbiacées - Loganiacées - Lythracées Burseracées.

\section{Anacardiacées}

Sclèrocarya birrhoea Hochst. - Heri, Hede (f). Feuilles et fruits consommés par bovins, ovins, caprins. Assez commun sur terres argileuses.

Lannea humilis. - Bellouki (f). Teuilles broutees en saison sèche par bovins, ovins, caprins. Commun en peuplements localisés sur terrains argileux (Mora).

\section{Anonacées}

Anona senegalensis Pers. - Dukuhi ladde (f). Jeunes feuilles consommées par chevaux, bovins, ovins. Commun en terrains alluvionnaires, sableux.

\section{Ampelidacées}

Ampelocissus grantii Planch. - Gubungaba (f). Racines lactogènes, feuilles fourragères. Assez commun.

Cissus populnea Guil. et Perr. - Gubuwol (f). Feuilles consommées par bovins, ovins, caprins. Assez commun.

\section{Asclepiadacées}

Leptadenia lancifolia Decne. - Zaraal (f). Feuilles et tiges consommćes par bovins, ovins, caprins. Espèce connue.

\section{Bignoniacées}

Kigelia aethiopica var. 'bornuensis Spragne.'Gillani (f). Feuilles fourragères, consommées par bovins, ovins, caprins. Commun en terres argileuses, bords de mares et rivières.

\section{Bombacées}

Adansonia digitata Linné, - Bokki (f). Feuilles comestibles et fourragères.

Bombax buonopozense P. Beauv: - Johi (f). Feuilles consommées par bovins, "ovins, caprins. Assez commun dans les massifs montagneux.

Ceiba pentandra Gaertn. - Bantahi (f). Kapokier forestier à graines déhiscentes. Le Service forestier a introduit une variété de 'Java' indéhiscente à la station agricole de Maroua en 1936. Feuilles fourragères en période de disette.

\section{Burseracées}

Commiphora africana. - Kabikonahi "(f). Jeunes repousses consommées par bovins, ovins, caprins en saison sèche. Assez commun terres de piémonts.

\section{Capparidacées}

Boscia senegalensis Lam. - Moheb (a), Buldumhi (f). Feuillage et fruits mangés par les chameaux (Mornet).

Cadaba farinosa Forsk. - Sireh (a), Senseni (f). Fourrager. Feuilles et fruits consommées par le bétail. Assez commun.

Capparis corymbosa Lam. - Feuillage fourrager et fruits.

Capparis decidua Pax. - Feuillage et fruits. 
Capparis tomentosa Lam. - Feuillage et fruits (chameaux).

Crataeva adansonii DC Holl. - (Samanaijko) (f). Feuillage.

Maerua angustifolia A. Rich. - Baguhelli (f).

Maerua angolensis DC Holl. - Buguhi (f).

\section{Combretacées}

Anogeissus schimperi Hochst. - Sahab (a), Kodjoli (f). Jeunes feuilles consommées par le bétail en période de disette (bovins, ovins, caprins). Petit arbre dans les boisements.

Combretum aculeatum Vent. - Lahugni (f). Feuilles appétées par le bétail (bovins, ovins, caprins). Arbuste commun au pied des massifs.

Guiera senegalensis Lam. - Abesh (a), Gelloki (f). Jeunes feuilles consommées par bovins, ovins, caprins, Assez commun sur les terrains alluvionnaires et sableux.

Terminalia avicenniö̈des Linné. - Saragayahi (f). Signalé au Niger comme consommé par les ruminants. Assez commun sur les terres alluvionnaires.

\section{Euphorbiacées}

Fluggea virosa Baill. - Karlje-Kartje (a), Tchammi (f). Arbuste très commun, buttes argileuses (termitières). Feuillage consomme par bovins, ovins, caprins.

\section{Légumineuses cesalpinées}

Afzelia africana Smith. - Petohi gayahi. Feuilles consommées par bovins, ovins, caprins. Peu commun, galeries forestières (Yagoua).

Bauhinia rufescens Lam. - Namarehi (f). Feuilles consommées par le bétail. Assez commun sur terres argileuses.

Daniellia oliveri Hutch. et Dalz. - Kaherlahi (f). Jeunes feuilles consommées par bovins, ovins, caprins. Galeries forestières, bords des mares (Yagoua).

Isoberlinia doka Craib et Stpaf. - Kubahi (f). Feuilles consommées par bovins, ovins, caprins. Massifs montagneux.

Parkinsonia aculeata Linné. - Gawari hidjou (f). Feuillage fourrager. Commun dans les villages.

Tamarindus indica Linné. - Jatami, Jabbi (f). Feuilles et fruits consommés par le bétail. Assez commun, terres profondes.

\section{Papilionacées}

Alysicarpus violaceus Schmidt. - Kongoladji (f): Plante semi-herbacée, consommée à l'état adulte, serait cultivée comme fourrage. Assez commune dans les terres cultivées.

Herminiera elaphroxylon Guill. et Perr. Tcharidji (1). Feuilles consommées par bovins, ovins, caprins. Prairies inondées, mares.

Lonchocarpus laxiflorus Guill. et Perr. - Raneranehi (f). Feuilles consommées par le bétail. Peu commun (massifs montagneux, galeries forestières)."

Pterocarpus erinaceus Poir. - Balebalehi (f), Banohi (f). Feuillage fourrager. Peu commun, galeries forestières. Souvent cultivé.

Sesbania divers. Feuilles consommées par bovins, ovins, caprins. Commun, prairies inondées.

\section{Légumineuses mimosées}

Cette grande famille est surtout représentée par les Acacia. Les autres genres fourragers sont :

Albizzia - Dichrostachys - Entada - Mimosa - Parkia - Prosopis.

\section{Acacias.}

Les acacias ont une grande importance au point de vue fourrager. Dans certaines régions (Sahara), ils constituent parfois l'unique pâture. Suivant leur aire écologique, on distingue :

\section{a) Acacias sahéliens.}

Acacia scorpioìdes (L) Chevalier. - Assez commun surtout aux bords des mares. Cette espèce est reconnaissable à son tronc noir, à écorce crevassée, à son port élevé, à ses gousses scorpioïdes.

Var. nilotica (gousses très étranglées). - Garad mermedi (a), Gaddi yaere (f).

Var. adstringens (gousses plus larges, noires, rétrécies), - Garad gabdi (a), Gawari gabdi ou gabde ( $\mathrm{f}$ ).

Acacia seyal. - Assez commun en tous terrains, surtout argileux.

Ce grand groupe d'acacias est reconnaissable à son tronc de couleur rougeâtre, jaunâtre ou verdâtre, à port bas, généralement à épines droites plus petites que chez $A$. sieberiana.

Il est désigné par les Arabes sous le nom de "dalhaya " et par les Foulbés sous le nom de "bulbi bodehi, tsilluki bodehi, tsilluki baleri (var. chariensis). "

Les feuilles de ce groupe sont fourragères; Hutchinson signale la vente des graines pour l'engraissement des moutons, chèvres.

Plusieurs sous-espèces :

Acacia flava (Forsk) Schweinf. var. camerunensis; Acacia stenocarpa Hochst. var. chariensis ;

Acacia seyal Del. 


\section{b) Acacias soudano-sahéliens.}

Faidherbia albida (Del) Chev. - C'est le meilleur fourrage. Ses fruits sont également appétés. Se distingue par ses feuilles d'un gris argenté qui tombent en saison des pluies, ses rameaux blanchâtres, ses epines, ses fruits crispés jaunes à maturité.

Araza, arazaya (a). Tchaski (f). Assez commun en terrain argileux et alluvions anciennes.

Acacia senegalensis Willd. - Patterlahi debbi (f), Al gittir (a). Reconnaissable à ses êpis de fleurs blanches parfumées et à ses 3 épines caracteristiques. Feuilles et fruits fourragers. Assez commun, surtout à Fort-Foureau.

Acacia sieberiana in Prod. II, 463 (1825). - Kub, kukaya (a), Alluki (f). Ce groupe présente de nombreuses variations écologiques. On le reconnaît à ses grandes gousses, à son tronc de couleur jaunâtre, à son port élevé, à ses grandes épines droites.

Acacia caffra Willd. var, campylacantha. $\Lambda$ shab (a), Pattuki (f). Le feuillago ne semble pas être appété par le bétail. Peu commun, bords des mares.

Acacia ataxantha D.C. - Abindru (a), Korahi (f). Acacia à feuillage fourrager (Chev.). Se distingue de A. macrostachya Reichenb. par la présence d'une glande stipitée et saillante à la base du pétiole. Cette glande est sessile chez $A$. macrostachya. Peu commun, galeries forestieres.

Acacia macrostachya. - Reichenb. Ex Benth. Gagorlahi (f), Feuilles consommées par les chèvres. Peu commun, galeries forestières.

Albizzia chevalieri Harms. - Aried, Derut (a). Fora fagnino (f). Feuillage signalé comme fourrager au Niger. Espèce peu ou pas appétée au Cameroun. Peu commun, galeries forestières.

Dichrostachys glomerata Chev. - Djiginjap (a), Saginahi (f). Feuillage et fruits fourragers. Assez commun.

Entada sudanica Schwein. - Ma fulfil (a), Fado wanduhi (f). Les feuilles et les fruits seraient consommés dans certaines régions par bovins, ovins, caprins. Surtout abondant plus au sud, Garoua, Adamaoua.

Mirnosa aspera Linné. - Amsinene (a), Gandjandji (f). Feuilles broutées par chèvres et moutons. Mares d'eau, berges inondées.

Prosopis africana Taub. - Kohi (f). Les feuilles sont consommées par les bovidés malgré la teneur en tanin. Assez commun, galeries forestières.

Parkia filicö̈dea Welw. - Narehi (f). Gousses signalées au Niger commes riches et appétées par le bétail. Peu commun, galeries forestières.

\section{Ioganiacées.}

Strychnos spinosa Lam. - Narbo tanahi (f) Feuilles consommées par bovins, ovins, caprins.

\section{Lythracées.}

Lawsonia inermis Linné. - Nalle (f). Feuilles consommées par bovins, ovins, caprins.

\section{Moracées.}

Ficus natalensis. - Tchekehi (f). Reconnaissable à ses feuilles et à ses fruits très petits. Feuilles consommées par le bétail. Commun dans les villages.

Ficus polita. - Litahi (f). Feuilles consommées par le bétail. Peu commun.

Ficus gnaphalocarpa. - Ibbi ouro (f). Feuilles consommées pendant la période de disette. Commun surtout en région sahélienne aux bords des mares et rivières.

Ficus thonningii. - Maliani (f). Feuilles consommées par le bétail. Commun dans les villages.

\section{Moringiacées.}

Moringa pterygosperma Gaertn. - Giligandjahi (f). Feuillage consommé par le bétail, arbuste cultivé.

\section{Rhamnacées.}

Zizyphus jujuba Lam. - Jabi (f). Forme sauvage $\mathrm{du}$ jujubier. Feuilles et fruits fourragers (chèvres).

Zizyphus spina christi Willd. - Kumahi (f). Feuilles et fruits fourragers.

\section{Rubiacées.}

Feretia canthioïdes Heim. - Gurgalhi (f). Arbuste à feuilles broutées par bovins, ovins, caprins. Pieds des massifs montagneux et terrains rocailleux. Peu commun.

Randia nilotica Stapf. - Burli danehi, burugalhi (f). Feuilles broutées par bovins; ovins, caprins. Assez commun, pieds des massifs, terrains rocailleux, argileux.

\section{Simarubacées.}

Balanites aegyptiaca Del. - Tanni (f). Pulpe des fruits et feuilles consommóes par le bétail.

\section{Tiliacées.}

Corchorus olitorius Linné. - Feuilles alimentaires et fourragères (chevaux, bovins, caprins, ovins).

Grewia cissolides Hutch. et Dalz. - Tchiboli (f). Feuilles consommées par les bovins, ovins, caprins. Commune.

Grewia mollis Juss. - Yayakelli (f). Feuilles consommées par bovins, ovins, caprins. Commune. 
Grewia villosa Willd. - Gursuhi, Gurfuhi (f). Feuilles consommées par bovins, caprins, ovins. Très commune.

\section{UImacées.}

Celtis integrifolia Lam. - Ganki (f). Feuillage fourrager ; assez commun, terres argileuses, piémonts.

\section{BIBLIOGRAPHIE}

AUBREVILLE (A.). - La Flore forestière soudanoguinéenne. Sociétés Éditions Maritimes et Coloniales, 17, rue Jacob, Paris, 1950.

- La végétation forestière des Monts Mandara. Bulletin Sc. no 2, p. 129. Publication de la S.T.A.T., 45 bis, avenue de la Belle-Gabrielle. Nogent-sur-Marne (Seine).

CHEVALIER (A.). - Plantes fourragères et graminées. Revue de Botanique Appliquée (années 1932 et suivantes), 57, rue Cuvier, Paris (Ve). DALZIEL (J.-M.). - The useful plants of West Tropical Africa. Londres, 1937.

HUTCHINSON et DALZIEL. - Flora of West Tropical Africa. Londres, 1936.
JACQUES-FÉlLIX (H.). - La Vie et la Mort du Lac Tchad. Bull. Agro. no 3, Mai 1947. Publication de la S.T.A.T. 45 bis, avenue de la BelleGabrielle, Nogent-sur-Marne (Seine).

MALZY (P.). - Quelques plantes du Nord-Cameroun et leur utilisation. Journal d'Agriculture Tropicale et de Botanique Appliquée. Vol. I, 1954, p. 149, 317-441.

MORNET (P.). - Ies arbres fourragers des zones sahéliennes et présahéliennes du Nigex. Niamey.

VAILLANT (A.). - Ia Flore mérldionale du Lac Tchad. Bull. Société Études Camerounaises, no 9, mars 1945.

Une enquête Agricole chez les Mofu de Wazam (Nord-Cameroun). Bull. Soc. Etudes Camerounaises, no 17, 18, mars, juin 1947. L'érosion du sol dans le Massif du Mandara (Nord-Cameroun). Communication à la conférence Africaine des Sols, Goma. Bull. Agricole Congo belge. 1949, vol. 2, p. 1243.

Cartes du Service Géographique au 1/200.000 sur Maroua - Yagoua - Fort-Foureau, indiquant le type de végétation [136 bis, rue de Grenelle, Paris (VIIe) ].

\section{SUMMARY}

\section{Natural pastures of the northern Cameroons}

The region studied is situated in the Northern Cameroons between the boundary line of the waters of the Benoue basin on the South, and Lake Chad on the North, the Mandara hills on the West and Logone on the East.

The writer sums up the climatic conditions rapidly, as well as the physical aspect of this zone. On the other hand, he expands upon the surface of the pasture lands, the possibility of their being over-stocked during certain seasons, the study of their improvement, and finally their composition according to the nature of the land.

The study of the principal species of gramineae used for fodder in this region is dealt with in the second part of the article.

\section{RESUMEN}

\section{Los pastos naturales del Cameroun-Norte}

La régión estudiada está situada en el Cameroun-Norte entre la linea de división de las aguas del valle de la Benoué en el sur, el lago Tchad en el norte, la cordillera montañosa del Mandara on el oeste y el Logone, en el este.

El autor pasa revista rápidamente al clima y al medio físico de esta zona. Se extiende, por e] contrario, en la superficie de los pastos, y su posible sobrecarga en ciertas estaciones, el estudio de su mejora y enfin, su composición según la naturaleza de los terrenos.

El estudio de las principales especies de gramíneas forrageras de esta región constituye la segunda parte del artículo. 